\title{
"No hacen viejos los años, sino los daños": envejecimiento y salud en varones rurales
}

\author{
V Nelly Salgado de Snyder, MA, PhD, (I) Tonatiuh T González-Vázquez, Lic en C Pol,(1) \\ Berenice Jáuregui-Ortiz, Lic en Pedag,(I) Pastor Bonilla-Fernández, M en C. (I)
}

\begin{abstract}
Salgado de Snyder VN, González-Vázquez TT, Jáuregui-Ortiz B, Bonilla-Fernández $\mathbf{P}$. "No hacen viejos los años, sino los daños": envejecimiento y salud en varones rurales. Salud Publica Mex 2005;47:294-302.
\end{abstract}

\begin{abstract}
Resumen
Objetivo.Analizar los factores sociales asociados al estado de salud de hombres mayores de 60 años que viven en contextos de pobreza en áreas rurales de México. Material y métodos. De febrero a octubre de 2002 se llevó a cabo un estudio transversal, descriptivo, con una muestra intencional de 392 adultos mayores residentes de zonas rurales de los estados de Guerrero, Morelos y Jalisco, utilizando un cuestionario especialmente diseñado y piloteado con anterioridad. Las variables de estudio incluyeron características sociodemográficas, bienestar generalizado, problemas de salud, consumo de algunos alimentos y algunos indicadores de salud mental, fuerza personal y apoyo social. Utilizando el paquete estadístico SPSS v. II.I se llevaron a cabo pruebas de ji cuadrada de Pearson para variables categóricas; para analizar el consumo diferencial de alimentos se hicieron pruebas de ANOVA de una vía. Resultados. Los ancianos de este estudio eran casados o viudos y tenían un promedio de seis hijos. Los ancianos de Morelos informaron haber padecido un mayor número de enfermedades y haber tenido un consumo más alto de bebidas alcohólicas, en comparación con los ancianos de los otros dos estados. De la muestra total, $60 \%$ notificaron el uso tanto de remedios caseros como de medicamentos para aliviar sus malestares de salud. Más de la mitad no recibía apoyo familiar adecuado. Conclusiones. Los resultados de este estudio sugieren que el proceso de envejecimiento entre los hombres participantes es percibido como una carga, fundamentalmente debido al contexto de pobreza en que viven. La falta de ingresos suficientes y acceso a servicios de salud a través de planes de pensión y jubilación adecuados para los ancianos que viven en pobreza, es un problema que requiere de atención inmediata.
\end{abstract}

Palabras clave: vejez; salud; México; pobreza; hombres

\author{
Salgado de Snyder VN, González-Vázquez TT, \\ Jáuregui-Ortiz B, Bonilla-Fernández P. \\ Ageing and health \\ among rural men. \\ Salud Publica Mex 2005;47:294-302.
}

\begin{abstract}
Objective. To analyze the social factors associated with the health status of elderly men, 60 years and older, who live in poverty in the rural areas of Mexico. Material and Methods. A cross-sectional, descriptive study was conducted between February and October 2002, in a convenience sample of 392 elderly men residing in rural areas of the states of Guerrero, Morelos and Jalisco states. A validated questionnaire was applied to collect data on sociodemographic characteristics, general wellbeing, health problems, food consumption, and some indicators of mental health, personal strength and social support. Data analysis was performed with the SPSS v. II.I software, to obtain chi-squared tests; food intake measures were analized with one-way ANOVA. Results. Study subjects were married or were widowers and had six children on average. A higher proportion of the elderly men of Morelos state reported to have suffered more illnesses and a higher consumption of alcohol when compared to the other two state groups. Sixty percent of the men in the study used both home remedies and medication for their health problems. More than one half of them did not receive adequate social support from their families. Conclusions. The results of this study suggest that the process of ageing among rural elderly men is perceived as a burden, mainly because of the context of poverty in which they live. The lack of appropriate income and access to health services through adequate pension and retirement plans for the elderly poor is a problem that requires immediate attention.
\end{abstract}

Key words: aging; health; Mexico; rural; poverty; men

Esta investigación se llevó a cabo con financiamiento del Programa Fulbright New Century Scholar (200I-2002) y de INDESOL, Programa de Investigación para el Desarrollo Local.

(I) Centro de Investigación en Sistemas de Salud, Instituto Nacional de Salud Pública. Cuernavaca, Morelos, México.

Fecha de recibido: I de diciembre de 2004 - Fecha de aprobado: 25 de mayo de 2005 Solicitud de sobretiros: Dra.V Nelly Salgado de Snyder. Dirección de Salud Comunitaria y Bienestar Social, Centro de Investigación en Sistemas de Salud, Instituto Nacional de Salud Pública. Avenida Universidad 655 colonia Santa María Ahuacatitlán 62502, Cuernavaca, Morelos, México.

Correo electrónico: nsnyder@insp.mx 
E envejecimiento es un proceso ineludible y gradual que se manifiesta, principalmente, en cambios morfológicos y fisiológicos como consecuencia de la acción del tiempo sobre los organismos vivos. ${ }^{1}$ En los seres humanos, este proceso invariablemente resulta en la manifestación y el aumento de problemas de salud, disminución de la independencia y adaptabilidad, deterioro de los modelos familiares y sociales, retiro del trabajo, merma en la capacidad económica y disminución de las facultades físicas y mentales. ${ }^{2}$

Se estima que en la actualidad existen 600 millones de ancianos en el mundo y se espera que para mediados del siglo esta cifra alcance los dos mil millones. ${ }^{3}$ En México, el envejecimiento se ha producido con gran velocidad: estadísticas recientes indican que en el año $2000,6.8 \%$ de la población eran adultos mayores ${ }^{*}, 4$, de acuerdo con las proyecciones, se espera que para el año 2050 la proporción aumente a 28\%. ${ }^{5}$ Si bien en nuestro país las estadísticas recientes indican que las condiciones de salud de los mexicanos en general han mejorado significativamente, el envejecimiento poblacional se ha convertido en un tema prioritario debido a que implica un aumento en la inversión de recursos sociales, económicos y humanos, así como en la planeación de programas y redefinición de prioridades en el área de la salud. Las proyecciones oficiales indican que en tan sólo 50 años, México alcanzará los niveles de envejecimiento que los países europeos han alcanzado en más de 200 años. ${ }^{6}$ El crecimiento vertiginoso de este grupo poblacional obliga a enfrentarse con problemas debidos a la falta de servicios institucionales adecuados para este grupo, y a que la misma sociedad carezca de la capacidad para ofrecerles la atención que requieren.?

México es un país de contrastes, con una composición étnica compleja; en términos de su distribución, la población urbana $(74.6 \%)$ se concentra en su mayoría en pocas ciudades, mientras que la rural (25.4\%) está dispersa en miles de comunidades con una densidad poblacional muy baja. ${ }^{8,9}$ Por ello, los esfuerzos de salud se han concentrado primordialmente en las áreas urbanas, donde reside la mayor cantidad de pobres, -a pesar de que la población rural se vea más afectada por la pobreza al carecer de muchos recursos que sí están disponibles para los habitantes de las ciudades-. ${ }^{10}$ Por ejemplo, la mayoría de los habitantes de las localidades rurales no tienen acceso a los servicios de salud

\footnotetext{
* De acuerdo con Instituto Nacional de las Personas Adultas Mayores, las personas adultas mayores en México son aquellas que cuentan con 60 años o más de edad y que se encuentren domiciliadas o en tránsito en el territorio nacional (referencia 4).
}

proporcionados en los centros urbanos por instituciones oficiales y de la sociedad civil. Muchos pobladores rurales se emplean en labores artesanales y en la agricultura de autoconsumo, por lo cual no tienen derechohabiencia. En muchas localidades rurales, médicos generales ofrecen sus servicios en consulta privada; $\sin$ embargo, esto representa un costo económico que la mayoría de las familias no alcanza a cubrir. ${ }^{11}$

Los adultos mayores en México tienden a concentrarse en los grupos sociales de menores ingresos. De los hogares con ancianos, 29\% se ubican en localidades rurales de menos de 2500 habitantes ${ }^{12}$ que se caracterizan por tener viviendas construidas con materiales frágiles y piso de tierra, además de otras carencias como falta de agua potable y energía eléctrica. ${ }^{13}$ Estas condiciones de pobreza, vulnerabilidad y limitada accesibilidad a servicios básicos se agudizan en la vejez.

En México hay más mujeres ancianas $(51.29 \%$ ) que hombres $(48.71 \%),{ }^{8}$ sin embargo, el índice de masculinidad entre los adultos mayores muestra una tendencia inversamente proporcional al tamaño de la localidad; es decir, en las localidades pequeñas o rurales hay más ancianos hombres que mujeres, y conforme aumenta el tamaño de la localidad se incrementa el número de mujeres. ${ }^{12}$ Es necesario tener presente la heterogeneidad en el proceso de envejecimiento, pues hay una gran diferencia entre la vejez masculina y la femenina ya que los roles de género tradicionales persisten hasta entonces. Son precisamente estos roles, en comunidades rurales de México, los que dictan que los varones manifiesten conductas machistas como aparentar fortaleza física o mental (por ejemplo, no expresar sufrimiento, ni dolor, no acudir al médico), mantener la autoridad y el poder en la familia y encargarse primordialmente de trabajar para llevar el sustento del hogar. ${ }^{14, *}$ Todas estas manifestaciones se ven afectadas durante la vejez debido al proceso natural de deterioro asociado a esta etapa.

Finalmente, los diferentes factores que determinan la calidad de vida en la vejez como el apoyo familiar, la salud, la economía, las relaciones afectivas, la viudez, etcétera, se van combinando, y permiten encontrar una amplia gama de formas de envejecer. ${ }^{15}$ Por ejemplo, el apoyo familiar es vital en el proceso de envejecimiento ya que éste ayuda a los adultos mayores a lidiar con sus enfermedades y problemas emocionales. Los hombres ancianos requieren más del apoyo familiar que las mu-

\footnotetext{
* DiGirolamo A, Salgado de Snyder VN. Women as primary caregivers in Mexico: Challenges to well-being. En: Nor Ba'yah AK, Kamsiah A, Ling HK, ed. Women and well-being. Sarawak: University of Malaysia. En prensa.
} 
jeres, debido a que la red social que construyen a lo largo de su vida suele estar basada principalmente en las relaciones que establecen en el ámbito laboral. ${ }^{16}$

En cuanto a padecimientos de salud mental, los pobladores rurales mexicanos presentan lo que culturalmente se conoce como "nervios". En los hombres, los "nervios" son causados, principalmente, por no cumplir con el rol tradicional de proveedores de la familia y se manifiestan por medio del consumo del alcohol, mismo que los lleva a comportarse de manera agresiva e irritable. ${ }^{14}$ En relación con los ancianos en México, existe escasa información y un inadecuado registro de los problemas de salud que no son causa de muerte o de hospitalización; ${ }^{17}$ el desconocimiento se agudiza al hablar de adultos mayores que no gozan de ningún apoyo institucional, sobre todo los que habitan en áreas rurales.

El objetivo de este estudio fue explorar, describir y analizar los factores sociales asociados con la salud física y mental de hombres mayores de 60 años de edad que viven en contextos de pobreza en áreas rurales de los estados de Guerrero, Morelos y Jalisco.

\section{Material y métodos}

Este fue un estudio transversal y descriptivo que incorporó dos aproximaciones metodológicas: cuantitativa y cualitativa. La parte cuantitativa se llevó a cabo con una muestra intencional de 604 ancianos y ancianas residentes de zonas rurales de los estados de Guerrero, Morelos y Jalisco. Los datos cualitativos fueron generados a través de entrevistas a profundidad con una submuestra $(n=38)$ de las personas ancianas encuestadas. Resultados parciales de los datos cualitativos* ${ }^{*}$ cuantitativos ${ }^{18}$ han sido analizados desde una perspectiva de género y se han notificado en otros trabajos.

Para este artículo se analizaron exclusivamente los datos cuantitativos de los participantes varones, ya que el número de ancianas fue casi la mitad $(n=203)$ del de los varones $(n=392)$. Finalmente, algunos testimonios derivados del abordaje cualitativo se utilizaron en el presente artículo únicamente con el propósito de ilustrar los resultados del estudio.

Las razones para elegir estos estados fueron, principalmente, por la diversidad de su grado de marginación a escala nacional. Según datos de Conapo, Guerrero ocupa el segundo lugar de marginación: $53.4 \%$ de su población vive en localidades menores a cinco mil ha-

\footnotetext{
* Treviño-Siller S, Pelcastre-Villafuerte B, Márquez-Serrano M. Experiencias de envejecimiento en el México rural: una perspectiva de género. (En proceso).
}

bitantes; además, 35\% de la población carece de drenaje y de sanitarios (porcentaje superior al de Chiapas, el estado con el mayor índice de marginación). Morelos tiene un grado de marginación medio: $23.9 \%$ de sus habitantes residen en localidades con menos de cinco mil habitantes. Por último, el grado de marginación de Jalisco es bajo y menos de $20 \%$ viven en localidades menores a cinco mil habitantes. ${ }^{19}$ Adicionalmente, en estos tres estados se observa de manera diferencial el proceso migratorio a Estados Unidos de América (EUA); así por ejemplo, Jalisco es un estado considerado de alta tradición migratoria, mientras que Morelos y Guerrero son de reciente incorporación a este fenómeno. La relación específica entre migración, vejez y bienestar se presenta en otro trabajo.*

Los participantes fueron seleccionados utilizando la técnica de "bola de nieve". Al hacer el contacto inicial se les explicó el objetivo y otros detalles del estudio asegurándoles confidencialidad y anonimato. Asimismo, se les pidió su consentimiento verbal informado para su participación de acuerdo con lo que establecen los principios éticos para las investigaciones con sujetos humanos de la Declaración de Helsinki. Además, el protocolo de investigación fue previamente aprobado por las tres Comisiones Evaluadoras del Instituto Nacional de Salud Pública (investigación, bioseguridad y ética).

El instrumento fue administrado por encuestadores con un nivel mínimo de estudios de licenciatura, quienes además fueron capacitados en un curso de 40 horas sobre el manejo y aplicación del mismo.

El cuestionario incorporó un total de 10 secciones; sin embargo, para el propósito del presente artículo, sólo se utilizaron algunas -características sociodemográficas, bienestar psicosocial, problemas de salud, consumo de alimentos, indicadores de salud mental, fuerza personal y apoyo familiar-, cuya mayoría contenía preguntas cerradas. Para evaluar el bienestar psicosocial se utilizó una escala de ocho reactivos que miden ese constructo de manera adecuada -por ejemplo: ¿qué tan satisfecho se siente acerca de sus éxitos y progresos logrados?; ¿qué tan seguro se siente de poder hacer frente a los problemas en el futuro?- La escala obtuvo un Alpha de Cronbach de 0.72 y el análisis factorial arrojó dos factores con valores eigen mayores de uno, los cuales en conjunto explicaron $48.2 \%$ de la varianza. El rango teórico de la escala fue de 8 a 24 .

La variable de fuerza personal se evaluó usando la escala del mismo nombre que desarrollaron Díaz Gue-

\footnotetext{
* González T, Jáuregui B, Bonilla P, Yamanis T, Salgado de Snyder VN. Wellbeing and familial support among Mexican elderly and its relationship to migration to the US. Documento no publicado.
} 
rrero y Melgoza. ${ }^{20}$ Dicha escala contiene cuatro reactivos que en esta muestra obtuvieron un alpha de Cronbach de 0.85 y un solo factor que explicó $69 \%$ de la varianza. Entre los ejemplos de los reactivos de esta escala se pueden citar aquí los siguientes: ¿qué tan fuerte se siente para cargar con su propia cruz? y ¿qué tan fuerte se siente usted para ayudar a sus familiares a cargar su cruz?, mismos que se responden en una escala tipo Likert de cuatro puntos que van de muy fuerte (4) a poco fuerte (1). El rango teórico de la escala fue de 4-16, en donde los puntajes altos sugieren mayor fuerza personal.

Finalmente, el apoyo familiar se evaluó mediante una escala de 12 reactivos -ejemplos: ¿qué tanto entienden su manera de pensar? y ¿cuánta confianza siente para platicar con ellos de sus problemas?- que se responden en una escala de cuatro puntos que van de nunca/nada (1) a siempre/mucho (4). En esta muestra de ancianos la escala de apoyo familiar percibido obtuvo un Alpha de Cronbach de 0.85 y en el análisis factorial se encontraron dos factores con valores eigen mayores a 1, que juntos explicaron $64 \%$ de la varianza.

Para conocer las diferencias estadísticas de algunas variables sociodemográficas y problemas de salud, así como del consumo de alcohol y tabaco entre los ancianos de Guerrero, Morelos y Jalisco, se llevaron a cabo pruebas de Ji cuadrada de Pearson para variables categóricas. Para analizar el consumo diferencial de alimentos se hicieron pruebas de ANOVA de una vía. Todos los análisis se realizaron usando el paquete estadístico SPSS v. 11.1 y con un nivel de significancia estadística de $95 \%$.

\section{Resultados}

Los participantes del estudio eran residentes de localidades rurales de menos de 5000 habitantes de los estados de Guerrero, Morelos y Jalisco. La mayoría de los ancianos declaró haber nacido en ranchos y pueblos pequeños (87.1\% de Jalisco, $96.4 \%$ de Morelos y 98.6\% de Guerrero) y haber pasado la mayor parte de su vida en esas comunidades, por lo que la muestra del estudio fue esencialmente rural. El cuadro I presenta las características sociodemográficas de los ancianos. El rango de edad de los participantes fue de 60 a 98 años, con un promedio de 71.1 años de edad. Se encontraron diferencias significativas en la edad de los tres grupos de ancianos: los de menor edad fueron los de Morelos, seguidos por los de Guerrero y Jalisco. Una proporción significativamente mayor de ancianos de Jalisco que de Guerrero y Morelos informó no tener escolaridad alguna $\left(r^{2}(2)=10.7 ; p<0.005\right)$, aunque la mayoría de ancianos de los tres grupos indicó saber leer y escribir.

En general, los ancianos participantes en el estudio eran casados o viudos y casi todos informaron tener descendencia, en promedio seis hijos. Sin embargo, los ancianos de Jalisco notificaron un mayor número de hijos que sus contrapartes de Morelos y Guerrero $(F(2,2)=5.22 ; p=0.006)$. En cuanto a su ocupación e ingresos, una proporción muy alta de ellos indicó que trabajaban por su cuenta o que eran empleados (63.5\%) y sólo una minoría dijo ser jubilado o pensionado. La jefatura de hogar en la mayoría de los casos recaía sobre los entrevistados, quienes en los tres estados indicaron vivir con su cónyuge (29.2\%); con sus hijos (8.7\%) o solos (14.6\%). Cabe destacar que se encontraron diferencias entre los grupos; así por ejemplo, una proporción significativamente más alta de ancianos de Jalisco que de los otros dos estados vivía con sus hijos, mientras que una proporción más elevada de morelenses que de los otros dos grupos notificó vivir solo $\left(r^{2}(8)=15.5 ; p<0.001\right)$. De forma similar, como se observa en el cuadro I, los de Guerrero y Morelos indicaron, con mayor frecuencia que los ancianos de Jalisco, vivir en casa propia $\left(r^{2}(4)=18.5 ; p<0.001\right)$ y trabajar por su cuenta $\left(r^{2}(6)=22.2 ; p<0.001\right)$.

Los ancianos de Guerrero informaron, en su mayoría, recibir apoyo económico o en especie de instituciones nacionales gubernamentales y no gubernamentales. Entre ellos también se encontró la proporción más baja de jubilados o pensionados (1.4\%). Esto contrasta con los ancianos de Jalisco, entre quienes una tercera parte indicó trabajar por su cuenta y una proporción similar respondió que eran jubilados o pensionados y que recibían estos fondos tanto de instituciones en México (66.7\%) como de EUA (33.3\%). En general esta muestra estuvo constituida por ancianos que vivían en pobreza rural.

La pobreza es más mala que el pecado mortal, porque la pobreza acarrea muchas dificultades, en lo físico, en lo personal, en lo moral (...). Usted sin dinero, ¿qué haría? ¿A quién le va a pedir 20 centavos o 20 pesos o mil pesos si nadie se los va a prestar porque lo ven jodido? Es allí en donde me entra el coraje y la muina. [Anciano jalisciense de 67 años (HNM-JAL-01)].

No hay corazón tan vacío como una bolsa sin dinero, aunque el dinero no es toda la felicidad... hay muchos medios de ser feliz. El dinero, si no lo sabe uno manejar, es la perdición, por eso es mejor no tenerlo. [Anciano jalisciense de 65 años (HM-JAL-02)]. 
Cuadro I

Características sociodemográficas

de los ancianos varones de Guerrero, Morelos y Jalisco. México, 2002

\begin{tabular}{lcccc}
$\begin{array}{l}\text { Características } \\
\text { sociodemográficas }\end{array}$ & $\begin{array}{c}\text { Guerrero } \\
(n=139)\end{array}$ & $\begin{array}{c}\text { Morelos } \\
(n=137)\end{array}$ & $\begin{array}{c}\text { Jalisco } \\
(n=116)\end{array}$ & $\begin{array}{c}\text { Total } \\
(n=392)\end{array}$ \\
$\begin{array}{l}\text { Edad promedio } \\
(D E)\end{array}$ & 71.4 & 69.6 & 72.6 & 71.1 \\
$\begin{array}{l}\text { Dónde nació (\%) } \\
\text { Rancho }\end{array}$ & $(7.7)$ & $(7.2)$ & $(8.0)$ & $(7.7)^{\ddagger}$ \\
\hline$\quad$ Pueblo & 12.9 & 32.8 & 38.8 & $27.6 *$ \\
\hline$\quad$ Ciudad & 85.6 & 63.5 & 48.3 & 66.8 \\
\hline & 1.4 & 3.6 & 12.9 & 5.6
\end{tabular}

Estado Civil (\%)

\begin{tabular}{lrrrr} 
Casado & 68.3 & 65.0 & 63.8 & 66.0 \\
\hline Viudo & 18.7 & 13.1 & 28.4 & 19.7 \\
\hline Vive con alguien & 4.3 & 10.9 & 0.9 & 5.6 \\
\hline Soltero/divorciado/separado & 8.7 & 10.2 & 6.9 & 8.6
\end{tabular}

Número de hijos $\bar{X}(D E)$

$5.8(2.6) 5.9(3.2) 6.9 \quad(3.1) 6.2 \quad(3.0)^{\ddagger}$

Nunca fue a la escuela (\%)

$\begin{array}{llll}38.1 & 32.8 & 52.6 & 40.6 *\end{array}$

Sabe leer (\%)

$\begin{array}{llll}65.5 & 74.5 & 75.0 & 71.4\end{array}$

Sabe escribir (\%)

$\begin{array}{llll}55.4 & 69.3 & 69.0 & 64.3 *\end{array}$

Condición de trabajo (\%)

\begin{tabular}{lrrrc} 
Empleado & 2.2 & 10.2 & 10.3 & $7.4^{*}$ \\
\hline Trabaja por su cuenta & 69.1 & 63.5 & 31.9 & 56.1 \\
\hline Jubilado/pensionado & 1.4 & 10.2 & 30.2 & 13.0 \\
\hline No trabaja & 27.3 & 16.1 & 27.6 & 23.5
\end{tabular}

Jefe de hogar (\%)

\begin{tabular}{lcrrc} 
Entrevistado & 94.2 & 83.9 & 73.0 & $84.4^{*}$ \\
\hline Cónyuge & - & 3.6 & 0.9 & 1.5 \\
\hline Hijos & 2.2 & 3.6 & 4.3 & 3.3 \\
\hline Otro & 3.6 & 2.2 & 8.7 & $4.6^{*}$ \\
\hline Entrevistado y cónyuge & - & 6.6 & 13.0 & 6.1
\end{tabular}

Con quién vive (\%)

\begin{tabular}{lrrrr} 
Cónyuge & 22.3 & 35.8 & 29.6 & $29.2^{*}$ \\
\hline Hijos & 4.3 & 5.8 & 17.4 & 8.7 \\
\hline Miembros de la familia & 22.3 & 19.0 & 23.5 & 21.5 \\
\hline Solo & 37.4 & 20.4 & 19.1 & 26.1 \\
\hline Amigos & 13.7 & 19.0 & 10.4 & 14.6
\end{tabular}

En dónde vive usted (\%)

\begin{tabular}{lrrrc} 
Cuarto rentado & 0.7 & 4.5 & 11.3 & $5.2^{*}$ \\
\hline Casa prestada & 7.2 & 6.0 & 21.7 & 11.1 \\
\hline Casa propia & 92.0 & 89.6 & 67.0 & 83.7
\end{tabular}

Recibe ayuda económica, pensión o jubilación (\%)

* Pruebas de ji cuadrada de Pearson, $p<0.05$

¥ Prueba de ANOVA de una vía, $p<0.05$
Cuadro II

Problemas de salud autonotificados

POR ANCIANOS DE GuERrERo,

Morelos y Jalisco. MÉXICO, 2002

Problemas

Guerrero Morelos Jalisco Total

de salud

$(n=139) \quad(n=137) \quad(n=116) \quad(n=392)$

Promedio de condiciones notificadas $3.0 \quad 4.1 \quad 3.0 \quad 3.4$

(DE)

$\begin{array}{llll}(1.9) \quad(2.5) \quad(2.3) \quad(2.3)^{\ddagger} & \end{array}$

\begin{tabular}{lcccc} 
& $\%$ & $\%$ & $\%$ & $\%$ \\
Artritis o reumatismo & $59.7^{*}$ & $44.5^{*}$ & $38.8^{*}$ & 48.2 \\
\hline $\begin{array}{l}\text { Presión arterial alta } \\
\text { Ceguera, sordera o impedimentos }\end{array}$ & 22.3 & 29.9 & 31.0 & 27.6 \\
\hline $\begin{array}{l}\text { severos } \\
\text { Nervios }\end{array}$ & $17.3^{*}$ & $36.5^{*}$ & $27.6^{*}$ & 27.0 \\
\hline
\end{tabular}

Problemas crónicos del estómago

como úlcera o colitis

I5. I $^{*} \quad 27.0 * \quad 24 . I^{*} \quad 21.9$

\begin{tabular}{lllll}
\hline Enfermedades de los riñones & $21.6 *$ & $29.2 *$ & $12.9 *$ & 21.7
\end{tabular}

\begin{tabular}{lllll}
\hline Asma, bronquitis o enfisema & $12.2 *$ & $23.4 *$ & $16.4^{*}$ & 17.3
\end{tabular}

Enfermedades relacionadas con

accidentes de trabajo

$\begin{array}{llll}20.9 & 19.0 \quad 11.2 \quad 17.3\end{array}$

Enfermedades de los dientes

\begin{tabular}{lllll}
\hline o la boca & $3.6 *$ & $26.3 *$ & $19.0 *$ & 16.1
\end{tabular}

\begin{tabular}{lllll}
\hline Diabetes & 10.1 & 15.3 & 19.8 & 14.8 \\
\hline
\end{tabular}

\begin{tabular}{lrrrr}
\hline Enfermedades de la piel & $5.0^{*}$ & $19.7^{*}$ & $12.1^{*}$ & 12.2 \\
\hline $18.0^{*}$ & $7.3^{*}$ & $7.8^{*}$ & 11.2
\end{tabular}

Ataques al corazón u otros

$\begin{array}{lllll}\text { problemas serios del corazón } & 6.5 & 9.5 & 15.5 & 10.2\end{array}$

\begin{tabular}{lllll}
\hline Susto & 6.5 & 10.2 & 6.9 & 7.9
\end{tabular}

\begin{tabular}{lllll}
\hline Problemas de la vesícula biliar & 3.6 & 9.5 & 5.2 & 6.1
\end{tabular}

Enfermedades relacionadas con

$\begin{array}{lllll}\text { el uso de pesticidas } & 4.3^{*} & 11.7^{*} & 1.7^{*} & 6.1\end{array}$

\begin{tabular}{lllll}
\hline Enfermedades del hígado & $2.2 *$ & $10.2 *$ & $5.2 *$ & 5.9
\end{tabular}

\begin{tabular}{lllll}
\hline Mal de ojo & $3.6^{*}$ & $8.8^{*}$ & $0.0^{*}$ & 4.3
\end{tabular}

\begin{tabular}{lllll}
\hline Embolia & I.4 & 4.4 & 3.4 & 3.1 \\
\hline
\end{tabular}

\begin{tabular}{lllll}
\hline Cáncer o tumor maligno & I.4 & 3.6 & 3.4 & 2.8
\end{tabular}

\begin{tabular}{lllll}
\hline Hepatitis & I.4 & 3.6 & 2.6 & 2.6
\end{tabular}

\begin{tabular}{lllll}
\hline Tuberculosis & 0.7 & 0.7 & 1.7 & 1.0
\end{tabular}

\begin{tabular}{lllll}
\hline Otro(s) & $43.9 *$ & $29.9 *$ & $13.8 *$ & 30.1
\end{tabular}

* Prueba de ji cuadrada de Pearson, $p<0.05$

¥ Prueba de ANOVA de una vía, $p<0.05$

\section{Salud física}

En el cuadro II se listan los 23 problemas de salud que se exploraron. Se encontraron diferencias significativas en la proporción de ancianos de los tres grupos que informaron haber padecido alguna enfermedad a 
lo largo de su vida $\left(r^{2}(2)=9.5 ; p<0.001\right)$. Llama la atención que los ancianos de Morelos notificaron haber padecido hasta ocho de las 23 condiciones de enfermedad exploradas, como bronquitis y enfermedades de las vías respiratorias $\left(r^{2}(2)=5.8 ; p=n s\right)$, impedimentos severos en la vista y audición $\left(r^{2}(2)=12.5 ; p=0.002\right)$, enfermedades de los riñones $\left(r^{2}(2)=9.6 ; p=0.008\right)$, del hígado $\left(r^{2}(2)=6.1 ; p=0.04\right)$, del estómago $\left(r^{2}(2)=6.1\right.$; $p=0.05)$ y de la piel $\left(r^{2}(2)=13.6 ; p=0.001\right)$. Asimismo informaron, en proporción mayor que los otros dos grupos, el padecimiento de enfermedades relacionadas con el uso de pesticidas $\left(r^{2}(2)=11.9 ; p=0.003\right)$. Finalmente, condiciones etnoculturales como "mal de ojo" $\left(r^{2}(2)=\right.$ 11.9; $p=0.003)$ y "susto" $\left(r^{2}(2)=1.5 ; p=n s\right)$, fueron, notificadas igualmente en mayor proporción por este grupo. Cabe destacar que los ancianos de Jalisco informaron padecer las proporciones más altas de diabetes $\left(r^{2}(2)=4.8 ; p=n s\right)$ y problemas cardiacos $\left(r^{2}(2)=5.8 ; p=n s\right)$. Por otra parte, una mayor proporción de los ancianos de Guerrero indicó haber padecido artritis $\left(r^{2}(2)=12.0\right.$; $p=0.002)$, "empacho" $\left(r^{2}(2)=10.0 ; p=0.007\right)$, enfermedades relacionadas con accidentes de trabajo $\left(r^{2}(2)=4.5\right.$; $p=$ ns) y otro tipo de enfermedades $\left(r^{2}(2)=27.2 ; p=0.000\right)$ que incluía, entre otras, epilepsia, trombosis, columna desviada, hernias y problemas de próstata.

Al hacer una sumatoria de las enfermedades que notificaron haber padecido a lo largo de su vida los ancianos de los tres estados, se observa que el promedio de enfermedades fue de $3.4(D E=2.3)$ y se detectaron diferencias significativas $(F(2,2)=9.5 ; p<0.001)$ entre los tres grupos de ancianos. Los morelenses obtuvieron el promedio más alto con 4.1 enfermedades $(D E=2.5)$; los de Guerrero y Jalisco alcanzaron un promedio de 3.0, con una $D E$ igual a 1.9 y 2.3 , respectivamente.

Ya me duele una cosa, ya me duele otra, tengo tres o cuatro enfermedades, tengo diabetes, enfermedad del cerebro, presión alta, gastritis. [Anciano morelense de 100 años (HNM-MOR-01)].

\section{Limitaciones asociadas a problemas de salud}

Una tercera parte de los participantes coincidió en que sus problemas de salud interferían con su trabajo (Guerrero $=35.3 \%$; Morelos $=29.9 \%$; Jalisco $=27.6 \%$; $p=n s$ ) y una menor proporción informó interferencia con su vida cotidiana (Guerrero $=11.7 \%$; Morelos $=11.5 \%$; Jalis$\mathrm{co}=17.2 \%$; $p=\mathrm{ns})$. De forma similar, se les preguntó si sus problemas de salud limitaban la cantidad de trabajo que podían hacer, y en sus afirmaciones se encontra- ron diferencias significativas (Guerrero $=38.1 \%$; Morelos $=46.7 \%$; Jalisco $\left.=30.4 \% ; r^{2}(2)=7.0 ; p<0.03\right)$. Al preguntar si los problemas de salud les impedían de alguna forma su funcionamiento, cerca de una tercera parte de los miembros de cada grupo contestó afirmativamente, sin llegar a obtenerse diferencias significativas entre los grupos (Guerrero=27.3\%; Morelos $=38.0 \%$; Jalisco $=32.2 \% ; p=n s)$.

Yo no tengo ni esperanzas de trabajar porque apenas puedo andar; eso sí me entristece, porque yo fui trabajador, fui arriero y además tenía una tierra. [Anciano morelense de 100 años (HNM-MOR-01)].

La tristeza viene con la vejez, ya se siente uno menos, ya inútil, pues ya no hace uno las cosas como antes; por ejemplo, yo ya no puedo montar a caballo, ya no puedo. [Anciano guerrerense de 78 años (HNM-GRO-01)].

Si me pongo a trabajar otra vez como trabajaba antes, le aseguro que en las primeras dos semanas saldría hasta con fiebre ... he sido tan pobre que he deseado un pan duro para comerme. [Anciano jalisciense de 64 años (HM-JAL-03)].

Es interesante mencionar que casi la mitad de cada grupo de ancianos contestó que sus problemas de salud no los habían limitado para realizar las actividades que la mayor parte de las personas de su edad son capaces de hacer (Guerrero 55.4\%; Morelos 49.0\%; Jalisco $53.4 \%$; $p=\mathrm{ns})$.

Sigo trabajando porque pienso que si no trabajo, pues no desquito lo que me voy a comer, porque lo dicen San Pedro y San Pablo: «el que no trabaje que no coma». [Anciano jaliscience de 65 años (HM-JAL-02)].

\section{Uso de medicamentos y remedios}

Una proporción elevada de los ancianos encuestados informó consumir remedios (Guerrero $=66.2 \%$; Morelos $=76.5 \%$; Jalisco $=64.7 \%$; $p=$ ns) y medicamentos (Guerrero $=63.8 \%$; Morelos $=73.0 \%$; Jalisco $=68.1 \%$; $p=n s$ ) para controlar y aliviar los malestares asociados con sus problemas de salud.

Ya tengo 12 años con una úlcera que apenas me la estoy aliviando; estuve con medicina de médicos y eso no me sirvió para nada, entonces me tiré a curarme con plantas de hierbas y eso y gracias a Dios que ya me estoy aliviando. [Anciano jalisciense de 84 años (HNM-JAL-04)] . 


\section{Consumo de alimentos}

Una sección del instrumento aplicado indagó acerca del consumo de algunos alimentos básicos en una semana típica (leche, carne, huevos, tortillas, chile, pan, frutas y verduras). Los resultados se encuentran en el cuadro III. Los ancianos de Guerrero notificaron el consumo más bajo $(p<0.01)$ de la mayoría de los alimentos incluidos en el cuadro como carne $(\mathrm{F}(2,388)=30.8)$ (pollo, res, cerdo o pescado), leche $(\mathrm{F}(2,386)=13.7)$, hue$\operatorname{vos}(F(2,389)=5.3)$, pan $(F(2,389)=10.0)$, frutas $(F(2$, $389)=30.6)$ y verduras $(F(2,389)=16.7)$. Como se señala en el cuadro, se encontraron diferencias significativas entre este grupo y los ancianos de Morelos y Jalisco. La falta de alimento se tradujo en algunos casos en delgadez extrema y debilidad.

[...] me canso mucho, yo ya no puedo caminar, llego aquí y me siento, por eso tengo dos cojines, pues las nalgas no tienen nada de carne, puros huesitos. Aquí me la paso sentado, no puedo hacer otra cosa. [Anciano guerrerense de 76 años (HM-GRO-02)].

\section{Consumo de alcohol y tabaco}

La mitad de los ancianos dijo fumar tabaco al momento de la entrevista (Guerrero $=52.6 \%$; Morelos $=40.2 \%$; Jalisco $\left.=43.5 \% ; r^{2}(2)=2.7 ; p=0.000\right)$. Sin embargo, los ancianos notificaron un bajo consumo de alcohol, ya que sólo una pequeña proporción informó beber "seguido" o "muy seguido" (Guerrero $=4.3 \%$; Morelos $=17.5 \%$; Jalisco=2.6\%; $\left(r^{2}(8)=22.9 ; p=0.000\right)$. No obstante, al profundizar en sus respuestas se encontró que una mayor proporción de los ancianos de Morelos

\section{Cuadro III}

Promedio de días de consumo de algunos alimentos EN UNA SEMANA TÍPICA, DE LOS ANCIANOS DE Guerrero, Morelos y Jalisco. Mexico, 2002

\begin{tabular}{lllll} 
Alimento & $\begin{array}{l}\text { Guerrero } \\
(n=\mid 39)\end{array}$ & $\begin{array}{l}\text { Morelos } \\
(n=\mid 37)\end{array}$ & $\begin{array}{c}\text { Jalisco } \\
(n=I I 6)\end{array}$ & $\begin{array}{c}\text { Total } \\
(n=392)\end{array}$ \\
Tortillas & $6.9(0.7)$ & $6.8(0.9)$ & $6.8(1.0)$ & $6.8(0.9)$ \\
\hline Chile/salsa & $6.2(1.9)^{*}$ & $5.4(2.6)$ & $4.2(3.0)^{*}$ & $5.3(2.6)$ \\
\hline Pan & $3.6(2.7)^{*}$ & $5.0(2.7)^{*}$ & $4.1(2.9)$ & $4.2(2.8)$ \\
\hline Leche & $2.9(3.0)^{*}$ & $4.2(3.0)$ & $4.8(3.0)^{*}$ & $3.9(3.0)$ \\
\hline Frutas & $2.0(2.1)^{*}$ & $4.2(2.7)^{*}$ & $4.2(2.8)^{*}$ & $3.4(2.7)$ \\
\hline Verduras & $2.2(2.0)^{*}$ & $3.4(2.7)^{*}$ & $3.9(2.8)^{*}$ & $3.1(2.8)$ \\
\hline Huevos & $2.2(2.0)^{*}$ & $3.0(2.3)^{*}$ & $2.5(2.4)$ & $2.6(2.3)$ \\
\hline Carne & $1.4(1.2)$ & $2.6(1.9)$ & $3.0(2.0)^{*}$ & $2.3(1.9)$
\end{tabular}

* Prueba de ANOVA de una vía, $p<0.05$ dijo beber tres o más copas por ocasión de consumo, de tal manera que su ingesta de alcohol era mayor a la de los otros grupos de ancianos (Guerrero $=53.1 \%$; Morelos $=77.9 \%$; Jalisco $=39.5 \% ; r^{2}(3)=18.6 ; p=0.000$ ).

\section{Salud mental}

En cuanto a los indicadores de salud mental, se utilizó una pregunta sobre el padecimiento de "nervios" y se encontró que los ancianos morelenses informaron en mayor proporción haber sufrido de "nervios" en algún momento de su vida (Guerrero=22.3\%; Morelos $=36.8 \%$; Jalisco $\left.=26.7 \% ; r^{2}(2)=7.2 ; p<0.02\right)$. Cabe destacar que los participantes que indicaron haber sufrido de "nervios" son aquellos que tienen un promedio de enfermedades más alto (5.4) $(D E=2.2)($ IC $95 \%=$ $2.55,3.0)$ que los que no han sufrido de nervios $(\bar{X}=2.8$ $(D E=1.9)(\mathrm{IC} 95 \%=4.9,5.8)$. Es importante hacer notar esta conexión entre salud física y salud mental entre los encuestados.

\section{Bienestar personal}

En relación con el bienestar personal, no se obtuvieron diferencias entre los tres grupos de ancianos (Guerrero: $\bar{X}=18.6$; Morelos: $\bar{X}=18.3$; Jalisco: $\bar{X}=18.3 p=n s$ ), pero sí una correlación entre los puntajes de la escala de bienestar personal y las enfermedades notificadas $(r=-0.22$; $p<0.001)$. La escala de fuerza personal ${ }^{20}$ obtuvo una media grupal de $9.8(D E=2.7)$, en donde los puntajes más altos fueron los de los ancianos de Jalisco $(\bar{X}=10.1$; $D E=2.7)$ y los más bajos correspondieron a los adultos mayores de Guerrero $(\bar{X}=9.2 ; D E=2.3)(F(2,2)=4.7$; $p<0.01)$. Se encontró una correlación inversa entre puntajes de bienestar personal y número de enfermedades $(r=-0.21 ; p=0.000)$.

\begin{abstract}
Dentro de toda mi enfermedad, pues me siento sano, bendito sea Dios, porque a la enfermedad hay que sufrirla con paciencia y con dignidad y dándole gracias a Dios por estar viviendo... soy pobre, pobre pero con felicidad. [Anciano jalisciense de 83 años con un pie amputado recientemente por gangrena (HM-JAL-05)].

La enfermedad es triste, cuando uno está enfermo hay que pedirle a Dios paciencia para tolerar las enfermedades que le mande a uno, porque ya sabe uno que se va a morir. [Anciano jalisciense de 65 años (HM-JAL-02)].
\end{abstract}

\section{Apoyo familiar}

Una de las variables que se evaluaron en este estudio fue el apoyo familiar percibido, medido con un instrumento especialmente diseñado para este propósito y 
cuyas características psicométricas se presentaron en la sección de material y métodos. La escala de apoyo familiar obtuvo un promedio grupal de 40.6 (DE=6.7), lo cual indica una percepción de apoyo familiar moderado; no se encontraron diferencias significativas entre los grupos. Empero, se observó una correlación inversa entre apoyo familiar y número de enfermedades $(r=-0.31 ; p=0.000)$. Específicamente, los ancianos que manifestaron que sus familiares discuten con ellos, los hacen sentir tensos, no se ocupan de ellos, no pasan tiempo con ellos y que sienten una menor satisfacción con el apoyo de la familia, fueron los que informaron un mayor número de enfermedades y una mayor prevalencia de "nervios".

Lo más difícil de hacerse viejo y ser pobre es que no haya hijos que estén a lado de uno, que no estén al cuidado de uno, que se vaya a topar uno con estar solo, que no tenga quién le ayude, quién le dé la mano. [Anciano jalisciense de 67 años (HNM-JAL-01)].

\section{Discusión}

Los datos que se presentan en este trabajo describen la situación de los adultos mayores que viven en la pobreza rural. Es evidente que los problemas de la vejez se agudizan cuando se vive con carencias económicas, sociales y personales, en especial en los varones que, contrariamente a las mujeres, tienden a envejecer en condiciones muy diferentes. ${ }^{21}$ Los resultados de este estudio revelan que poco más de la mitad de los participantes vivía con su pareja o algún otro miembro de la familia y el resto vivían solos o con amigos, a pesar de que la mayoría de los ancianos tenían un promedio de seis hijos. La mayoría de los hombres dijeron ser el jefe de su hogar y trabajar por su cuenta, aunque con limitaciones, lo cual obedece tanto a la pobreza en la que se encuentran como a los roles de género tradicionales que prevalecen en las comunidades rurales.

Se observó que los hombres ancianos notificaron en promedio tres enfermedades de un total de 23 listadas; es posible que el bajo reporte responda, entre otros factores, a la ignorancia de síntomas; al no acceso a los servicios de salud (por falta de información o de recursos económicos o por la ausencia de tales servicios en sus localidades); a la ausencia de una red de apoyo o al uso de servicios informales (curandero, sobador, yerbero, etcétera). De hecho se hizo evidente una correlación inversa de bienestar y apoyo social con el número de enfermedades reportadas. Todos estos factores han sido documentados como posibles barreras a la utilización de servicios formales de salud en las comunidades rurales de México. ${ }^{22}$ En el caso particular de los varones, la no utilización de servicios de salud puede estar asociada con los roles de género tradicionales que dictan que los hombres deben "aguantar" y no expresar sufrimiento, dolor o vulnerabilidad alguna. ${ }^{14}$ Otra razón puede ser la ausencia de derechohabiencia, aunque no se investigó esta variable.

Igualmente vinculado con los roles de género tradicionales, se encontró que la mayoría de los ancianos encuestados continúan trabajando a pesar de que muchos tienen un estado de salud precario y problemas que limitan severamente o impiden sus actividades cotidianas. Esta característica prevaleció sobre todo entre los ancianos más pobres, es decir aquellos de Guerrero y Morelos. A pesar de que la mayoría recibía ayuda del gobierno - principalmente del Programa Oportunidades-, al parecer ésta era insuficiente para cubrir sus necesidades básicas, como la alimentación, de tal manera que estaban obligados a continuar trabajando. La mitad de los ancianos de Jalisco no trabajaba porque recibían ingresos de su jubilación o pensión de EUA -la mayoría dijo haber trabajado en ese país-, o porque estaban incapacitados y tenían familiares que se hacían cargo de ellos. Este hallazgo confirma que los ingresos económicos y el apoyo familiar percibido son factores importantes para envejecer con calidad de vida. La falta de un plan adecuado de pensiones y jubilaciones para los ancianos pobres en México es un problema que debe ser atendido de manera inmediata por las autoridades, sobre todo porque las proyecciones demográficas indican claramente un aumento considerable de este grupo poblacional tan vulnerable. ${ }^{7}$

La alimentación de los ancianos de Guerrero fue menos variada, ya que estuvo basada primordialmente en el consumo de tortillas y chile; a diferencia de los ancianos de Jalisco y Morelos, quienes notificaron el consumo de otros alimentos como carne, huevos, pan, leche, frutas y verduras. De nueva cuenta, la precaria situación económica de los ancianos dificulta que consuman los alimentos necesarios para tener una dieta balanceada.

Paradójicamente, los ancianos de Guerrero informaron haber hecho un mayor consumo de tabaco, y los de Morelos una ingesta de alcohol mucho mayor que los otros dos grupos de ancianos. Esto puede estar relacionado con varios factores que requieren ser investigados, por ejemplo: la influencia del turismo y las actividades recreativas que involucran el consumo de alcohol en el estado de Morelos; las costumbres locales asociadas con los roles de género tradicionales asignados a los hombres; la ausencia de grupos de autoayuda como Alcohólicos Anónimos; la producción casera de bebidas alcohólicas y la ingestión de alcohol entre los varones como automedicación o mecanismo para enfrentar problemas, sobre todo emocionales. Esta última conclusión llama la atención en vista de que en 
los ancianos de Morelos también se presenta la prevalencia más alta de "nervios".

Es interesante observar que, a pesar de todas las condiciones adversas que enfrentan, la mayoría de los ancianos de los tres grupos -sobre todo los hombres de Guerrero- obtuvieron niveles altos de bienestar y de fuerza personal, medidas que reflejan la capacidad para enfrentar problemas propios, de la familia y de los amigos. Esta aparente fortaleza puede estar asociada nuevamente con los roles de género que dictan que los hombres deben ser fuertes. Finalmente, el apoyo familiar que perciben los ancianos encuestados indica que éste es inadecuado para la mayoría; es decir, no cuentan con los recursos familiares que les ayuden a sobrellevar los problemas que enfrentan en su vejez, llevándolos a pasar sus últimos años solos y, en ocasiones, abandonados. Las razones pueden estar vinculadas con la pobreza que afecta a todo el grupo familiar o con la ausencia de este último, ya que se ha documentado que en las zonas rurales empobrecidas, los grupos poblacionales más jóvenes tienden a salir de sus comunidades a las ciudades o hacia EUA en búsqueda de un trabajo que les garantice un ingreso suficiente para por lo menos cubrir las necesidades básicas de su familia. ${ }^{23}$

Los resultados de este artículo ofrecen un panorama general acerca del proceso de envejecimiento masculino en las zonas rurales de México. Ese proceso se vive como una carga más que los ancianos deben enfrentar debido, fundamentalmente, a la falta de recursos económicos y familiares que les aseguren llegar a esta etapa de la vida en condiciones dignas. La ausencia de ingresos adecuados, el insuficiente acceso a los servicios de salud y la escasez de programas de jubilación y pensiones en las zonas rurales es un problema que debe atenderse de forma prioritaria.*

\section{Agradecimientos}

Agradecemos los comentarios a versiones anteriores de este trabajo de la doctora Annette Fitzpatrick y la maestra Nina Yamanis.

\section{Referencias}

I. León V, Zunzunegui MV, Muñoz PE, Soria C. Contactos sociales y deterioro cognitivo en personas mayores de 65 años. Rev Gerontol 1991;1:105-110.

\footnotetext{
* La necesidad de atender éstos y otros problemas de la vejez en el medio rural han sido documentados en el volumen ya referido editado por Salgado de Snyder y Wong, 2003.
}

2. Ham R. Conceptos y significados del envejecimiento en las políticas de población. En: Consejo Nacional de Población. Envejecimiento demográfico de México: retos y perspectivas. México: Consejo Nacional de Población; 1999:41-53.

3. Organización Panamericana de la Salud. Envejecimiento: ¿qué nos depara el futuro? Comunicado de prensa. Organización Panamericana de la Salud, enero 2002; vol. 0I. Disponible en: http://www.paho.org/ Spanish/DPI/I00/I00feature02.htm [2005 febrero I0].

4. Secretaría de Desarrollo Social/Instituto Nacional de las Personas Adultas Mayores. Ley de los Derechos de las Personas Adultas Mayores. México, 2002.

5. Consejo Nacional de Población. Proyecciones de la población de México, 2000-2050. México: Consejo Nacional de Población; 2002. 6. Fondo de Población de las Naciones Unidas. Estado de la población mundial 1998. Nueva York: Fondo de Población de las Naciones Unidas; 1998.

7. Knaul F,Arreola H, Borja C, Nigenda G, Ruiz de Chávez M, Soberón G. Competitividad y salud: la propuesta. México, DF: Fundación Mexicana para la Salud; 2004.

8. Ham R. El envejecimiento en México: El siguiente reto de la transición demográfica. México, DF: El Colegio de la Frontera Norte- Miguel Ángel Porrúa; 2003.

9. Instituto Nacional de Estadística, Geografía e Informática. XII Censo General de Población y Vivienda, 2000.Tabuladores Básicos.Aguascalientes: Instituto Nacional de Estadística, Geografía e Informática; 2001.

10. Salles V. Pobreza, pobreza y más pobreza. En: Szasz I, Salles V, ed. Las mujeres en la pobreza. México, DF: El Colegio de México; 1994:47-72. II. Salgado-de Snyder VN, Díaz-Pérez MJ, Maldonado M, Bautista E. Pathways to mental health services among inhabitants of a Mexican village with high migratory tradition to the United States. Health Soc Work 1998;24:136-148.

12. Negrete M. Distribución geográfica de la población mayor. DEMOS 200I; I4:18-20.

13. Tuirán R. Familia y valores: cambios y arraigos tradicionales. DEMOS 1995;8:30-31.

14. Salgado de Snyder VN, Díaz-Pérez MJ. La salud mental de las mujeres rurales: problemas, necesidades y alternativas. En: Lara MA, Salgado de Snyder VN, ed. Cálmese son sus nervios, tómese un tesito: la salud mental de las mujeres mexicanas. México, DF: PAX; 2002:I7I-194. 15. Reyes L. El envejecimiento de la población zoque de Chiapas. DEMOS 200I; I4: 29-30.

16. Scott A, Wenger $C$. Género y redes de apoyo social en la vejez. En: Arber S, Ginn J, coord. Relación entre género y envejecimiento. Enfoque Sociológico. Madrid: Nardea; 1996.

17. Gutiérrez L. El proceso de la transición epidemiológica. DEMOS 2001;14:8-9.

18. Salgado de SnyderVN. Envejecimiento, género y pobreza en México rural. En: Salgado de Snyder VN, Wong R, ed. Envejeciendo en la pobreza: género, salud y calidad de vida. Cuernavaca: Instituto Nacional de Salud Pública, 2003:37-56.

19. Consejo Nacional de Población. Indices de marginación, 2000. México: Consejo Nacional de Población; 2001.

20. Díaz-Guerrero R, Melgoza E. Fuerza personal. Medida breve de fibra emocional. Aletheia 1994;13:21-24.

2I. Salgado de Snyder VN, Wong R, ed. Envejeciendo en la pobreza: género, salud y calidad de vida. Cuernavaca: Instituto Nacional de Salud Pública; 2003.

22. Salgado de Snyder VN, Díaz-Pérez MJ, González-Vázquez T. Modelo de integración de recursos para la atención de la salud mental en la población rural de México. Salud Publica Mex 2003;45:19-26. 23. Salgado de Snyder VN.Vejez y pobreza en México: conclusiones y retos. En: Salgado de Snyder VN,Wong R, ed. Envejeciendo en la pobreza: género, salud y calidad de vida. Cuernavaca: Instituto Nacional de Salud Pública; 2003:199-205. 